\title{
Gastric Varices with Remarkable Collateral Veins in Valpronic Acid-Induced Chronic Pancreatitis
}

\author{
Y. Hattori ${ }^{\mathrm{a}}$ T. Higurashi ${ }^{\mathrm{b}} \quad$ H. Takahashi ${ }^{\mathrm{a}} \quad$ M. Inamori $^{\mathrm{a}}$ \\ Y. Abe ${ }^{\text {a }}$ Y. Rino ${ }^{c}$ A. Nakajima ${ }^{a}$ \\ ${ }^{a}$ Gastroenterology Division, Yokohama City University Graduate School of \\ Medicine, ${ }^{b}$ Gastroenterology Division, Yokohama Rosai Hospital, and 'Department \\ of Surgery, Yokohama City University Graduate School of Medicine, Yokohama, \\ Japan
}

\section{Key Words}

Gastric varices · Valpronic acid · Chronic pancreatitis .

\begin{abstract}
Valproic acid (VPA) is a commonly prescribed and approved treatment for epilepsy, including Angelman syndrome, throughout the world. However, the long-term administration of drugs like VPA is associated with the possible development of gastric varices and splenic obstruction as a result of chronic pancreatitis. Such cases can be difficult to treat using endoscopy or interventional radiology because of hemodynamic abnormalities; therefore, surgical treatment is often necessary.
\end{abstract}

\section{Introduction}

Angelman syndrome is characterized by the deletion of $15 \mathrm{q} 11.2-\mathrm{q} 13$ and is characterized clinically by psychomotor retardation, a happy disposition with paroxysms of laughter, and epileptic seizures [1]. Valproic acid (VPA) is often prescribed for the epileptic seizures associated with this syndrome. Although VPA-induced pancreatitis is relatively uncommon, serious adverse effects - including diabetes, pancreatic cancer and pseudocysts - have been reported. Splenic vein obstruction can also arise as a complication of chronic pancreatitis $[2,3]$. Here, we report a case with remarkable collateral veins caused by splenic vein obstruction associated with chronic pancreatitis. 


\section{Case Report}

A 36-year-old man had been followed for VPA-induced chronic pancreatits for 17 years, and intravenous hyperalimentation nutrition had been administrated. In 2006, the patient presented at the emergency department with upper gastrointestinal bleeding. Abdominal CT and CT angiography showed multiple pancreatic calculi, splenic vein obstruction, and perigastric and subphrenic dilated collateral veins into the superior mesenteric vein (fig. 1). An upper gastrointestinal endoscopy revealed stomach varices ( $\mathrm{Lg}-\mathrm{f}, \mathrm{F} 2, \mathrm{RC}+$ ) on the posterior wall of the gastric body (fig. 2). Gastric varices caused by splenic vein obstruction as a result of chronic pancreatitis were diagnosed, and these varices were thought to be the cause of the upper gastrointestinal bleeding. Endoscopic treatment and catheterization with balloon-occluded retrograde transvenous obliteration were difficult to perform because of the Lg- $f$ varices, and no vein approach from the inferior vena cava was available; therefore, surgical treatment was indicated. A total gastrectomy, splenectomy, and enterostomy were performed, and the patient has not experienced any further episodes of bleeding since the operation.

\section{Discussion}

Although VPA-induced pancreatitis is very rare, it has been estimated to occur in $1: 40,000$ patients [4]. It is a fact that many causes exist for pancreatitis, however VPA is most suspected as a cause of pancreatitis in this case. Furthermore, it is suggested that numerous pancreatic complications and/or pancreatitis-associated diseases may affect the course and determine the prognosis of chronic pancreatitis, and biological markers and/or imaging procedure development were needed to detect chronic pancreatitis at its reversible stage $[5,6]$. Thrombosis of the splenic vein has been reported in $7-20 \%$ of patients with chronic pancreatitis. Additionally, bleeding occurs in approximately 5-10\% of patients with chronic pancreatitis [7-9]. The causes of gastrointestinal bleeding associated with chronic pancreatitis include splenic vein obstruction, sinistral portal hypertension, rupture of a pseudoaneurysm, bleeding from a pseudocyst, and peptic ulcer arising from chronic alcohol use or the loss of exocrine function [10]. Splenic obstruction may result from the following $[11,12]$ : (1) extrinsic compression by a pseudocyst, (2) fibrosis of the pancreatic parenchyma, and (3) injured endothelia and thrombosis as a result of perivenous inflammation from pancreatitis. In patients with cirrhosis, the collaterals are hepatofugal; this often leads to the formation of gastroesophageal varices. In contrast to generalized portal hypertension, the collaterals are hepatopetal in splenic vein thrombosis. Splenoportal collaterals decompress the short gastric veins through both the gastric coronary vein into the portal vein and via the gastroepiploic arcade into the superior mesenteric vein $[13,14]$. Splenic vein obstruction leads to isolated varices in the fundus of the stomach or hypersplenism, without associated varices in the esophagus. New therapeutic agents have been shown to retard the progression of chronic pancreatitis in a mouse model and may be useful in the treatment of drug-induced pancreatitis [15]. If the long-term administration of a drug like VPA is needed, the possibility of gastric varices or splenic obstruction arising from chronic pancreatitis should be considered. 
Fig. 1. CT angiography showing multiple pancreatic calculi, splenic vein obstruction, and perigastric and subphrenic dilated collateral veins into the superior mesenteric vein.

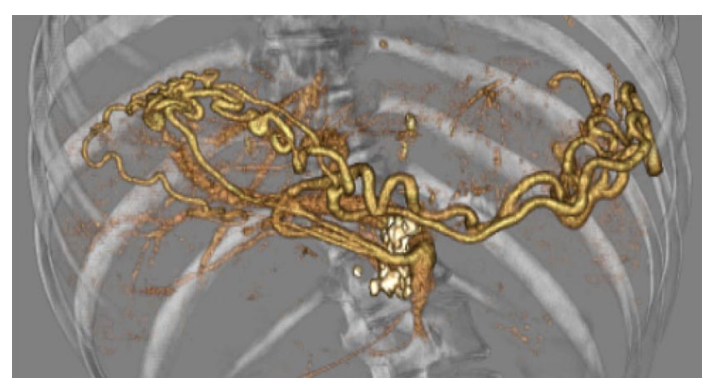

Fig. 2. Upper gastrointestinal endoscopy revealing stomach varices ( $\mathrm{Lg}-\mathrm{f}, \mathrm{F} 2, \mathrm{RC}+)$ on the posterior wall of the gastric body.

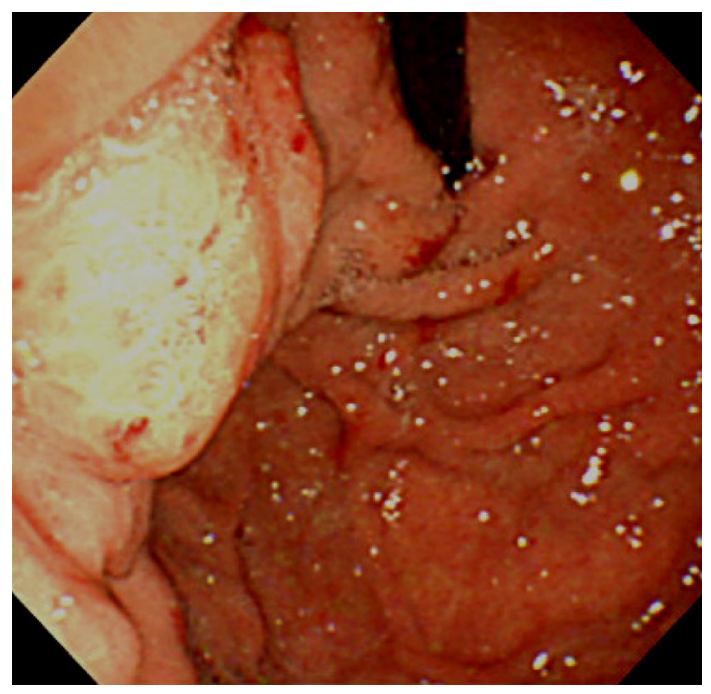




\section{References}

-1 Williams CA, Beaudet AL, Clayton-Smith J, Knoll JH, Kyllerman M, Laan LA, Magenis RE, Moncla A, Schinzel AA, Summers JA, Wagstaff J: Angelman syndrome 2005: updated consensus for diagnostic criteria. Am J Med Genet A 2006;140:413-418.

-2 American Academy of Family Physicians: Chronic pancreatitis: what you should know. Am Fam Physician 2007;76:1693-1694.

-3 Singhal D, Kakodkar R, Soin AS, Gupta S, Nundy S: Sinistral portal hypertension. A case report. JOP 2006;7:670-673.

-4 Gerstner T, Büsing D, Bell N, Longin E, Kasper JM, Klostermann W, Hebing B, Hanefeld F, Eckel U, Hoffmann R, Bettendorf U, Weidner B, Wiemer-Kruel A, Brockmann K, Neumann FW, Sandrieser T, Wolff M, König S: Valproic acid-induced pancreatitis: 16 new cases and a review of the literature. J Gastroenterol 2007;42:39-48.

5 Otsuki M: Chronic pancreatitis. The problems of diagnostic criteria. Pancreatology 2004;4:28-41.

6 Lankisch PG: Natural course of chronic pancreatitis. Pancreatology 2001;1:3-14.

7 Schwartz SA, Stubbs AY, Taljanovic MS, Smyth SH: Pancreatitis-associated splenic vein thrombosis with intrasplenic venous thrombosis: a case report. Emerg Radiol 2008: Epub ahead of print.

8 Heider TR, Azeem S, Galanko JA, Behrns KE: The natural history of pancreatitis-induced splenic vein thrombosis. Ann Surg 2004;239:876-880.

-9 Sakorafas GH, Sarr MG, Farley DR, Farnell MB: The significance of sinistral portal hypertension complicating chronic pancreatitis. Am J Surg 2000;179:129133.

10 Kaurich T: Drug-induced acute pancreatitis. Proc (Bayl Univ Med Cent) 2008;21:77-81.

11 Hall RI, Lavelle MI, Venables CW: Chronic pancreatitis as a cause of gastrointestinal bleeding. Gut 1982;23:250-255.

12 Cakmak O, Parildar M, Oran I, Sever A, Memis A: Sinistral portal hypertension; imaging findings and endovascular therapy. Abdom Imaging 2005;30:208-213.

13 Weber SM, Rikkers LF: Splenic vein thrombosis and gastrointestinal bleeding in chronic pancreatitis. World J Surg 2003;27:1271-1274.

14 Madsen MS, Petersen TH, Sommer H: Segmental portal hypertension. Ann Surg 1986;204:72-77.

15 Talukdar R, Saikia N, Singal DK, Tandon R: Chronic pancreatitis: evolving paradigms. Pancreatology 2006;6:440-449. 